\title{
¿Posee la ética un fundamento objetivo? Reflexiones desde Wittgenstein sobre el problema de explicar los juicios de valor ${ }^{1}$
}

\author{
Does ethics have an objective basis? Reflections from \\ Wittgenstein on the problem of explaining the value \\ judgments \\ Recibido: 10 de febrero de 2015 - Revisado: 26 de junio de 2015 - Aceptado: 13 de octubre de 2015
}

Jean Paul Sarrazin ${ }^{2}$

\begin{abstract}
Resumen
En este artículo recurrimos a diferentes aportes de Ludwig Wittgenstein (fuentes primarias y secundarias) con el fin de analizar críticamente ideas comunes sobre los orígenes y fundamentos de nuestros juicios éticos. Para esto, argumentamos que la distinción que hace el filósofo entre razones y causas es pertinente para demostrar que las valoraciones éticas no tienen un cimiento natural ni objetivo. Las valoraciones, en cambio, se apoyan en razones que surgen de un sistema sociocultural y se constituyen a través del lenguaje. Así, llegamos a plantear que el concepto de "juegos de lenguaje" propuesto por Wittgenstein, permite comprender mejor la forma en que los individuos interiorizan reglas y criterios a partir de los cuales realizan juicios éticos y establecen principios morales.
\end{abstract}

\section{Palabras clave}

Juicios éticos, explicaciones, Wittgenstein, valores morales, lenguaje.

\begin{abstract}
In this article we use different contributions of Ludwig Wittgenstein (primary and secondary sources) in order to critically analyze common ideas about the origins and foundations of our ethical judgments. For this, we argue that the distinction made by the philosopher between reasons and causes is relevant to show that ethical values have no natural or objective foundation. Valuations, however, rely on reasons arising from a sociocultural system and are constituted through language. Thus, we propose that the concept of "language games" proposed by Wittgenstein, can better allow an understandimg of how individuals internalize rules and criteria from which ethical judgments are made and moral principles are established.
\end{abstract}

\section{Keywords}

Ethical judgements, explanations, Wittgenstein, moral values, language.
${ }^{1}$ Este artículo procede del trabajo de investigación independiente del autor, sin financiación institucional. ${ }^{2}$ Antropólogo con opción en Filosofía por la Universidad de los Andes, Bogotá, Colombia. Magíster en Migraciones y Relaciones Interétnicas por la Université de Poitiers, Poitiers, Francia. Doctor en Sociología por la misma institución. Docente-investigador de planta vinculado al Departamento de Sociología de la Universidad de Antioquia.

Correo electrónico: jpsarra@yahoo.com

Para citar este artículo use: Sarrazin, J. (2015). ¿Posee la ética un fundamento objetivo? Reflexiones desde Wittgenstein sobre el problema de explicar los juicios de valor. Revista Civilizar Ciencias Sociales y Humanas, 15(29), 215-226. 


\section{Introducción}

El tema de la ética fue mencionado por el "primer Wittgenstein", en específico en su Tractatus logico-philosophicus (Wittgenstein, 2009) y en la "Conferencia sobre Ética" (Wittgenstein, 1965). El "segundo Wittgenstein" muy poco abordó directamente el concepto, aunque sí analizó las cuestiones estéticas, las cuales, como veremos, vinculó de algunas maneras a las cuestiones éticas. Así, el filósofo hizo afirmaciones como: "siempre he deseado decir algo sobre la gramática de las expresiones éticas o, por ejemplo, la palabra Dios" (Moore, 1983 , p. 357, citando una conversación con Wittgenstein).

Aunque el problema de explicar una valoración ética no fuera tratado en extenso, esto no se debe a que lo considerara nimio, sino porque lo incluyó en un dominio más amplio del pensamiento, en particular el del lenguaje, y en un estudio más general sobre los juicios de valor de todo tipo, tanto éticos como estéticos. "Lo que trató con cierta extensión no fue la ética, sino la estética, diciendo por ejemplo, 'prácticamente todo lo que digo sobre bello se aplica de un modo ligeramente distinto a bueno"' (pp. 357-358).

La presente reflexión comienza con una revisión del modo en que el primer Wittgenstein consideró la cuestión ética y su relación con el lenguaje, para luego concentrarnos en los aportes del filósofo austríaco en su segunda etapa, analizando sobre todo la división que él establece entre las razones y las causas en la explicación de los fenómenos socioculturales y la crítica que se infiere de allí a propósito del "naturalismo"; posteriormente se examinan sus reflexiones respecto del lenguaje, llegando al concepto de "juegos de lenguaje", el cual es útil para explicar los juicios éticos.

Tradicionalmente, una "investigación sobre el bien", implicaría que: a) “el bien”, en tanto que concepto general, es una suerte de contenido mental, b) basado en las cualidades intrínsecas de los objetos calificados y c), que aclarar el origen de una valoración ética es algo similar a explicar un fenómeno fisiológico o cerebral. En un acercamiento muy diferente, el segundo Wittgenstein nos insta a pensar la cuestión de los juicios éticos desde su fundamento en el lenguaje, recordando que este es una construcción intersubjetiva que se apoya en convenciones arbitrarias.

El adjetivo "arbitrario" -que utilizaremos con frecuencia y de manera conscientemente provocadora- no debe entenderse como sinónimo de "caprichoso" o "azaroso", ni se debe relacionar con un "acto contrario a la justicia, la razón o las leyes" (como el Diccionario de la Real Academia de la Lengua define la palabra "arbitrariedad"). Despojándolo de toda connotación negativa, entendemos el término como aquello que surge de la voluntad humana.

Al escoger este vocablo, nos basamos principalmente en el célebre lingüista Ferdinand de Saussure, quien afirmó que el lazo que une al significante con su significado es arbitrario (Saussure, 1945, p. 93), es decir, que los signos que conforman los lenguajes son arbitrarios. Por ejemplo, significantes como "tree", "arbre" o "árbol", usados para designar un objeto, no surgen de la naturaleza del objeto designado, y por eso mismo podemos decir que el signo no tiene un fundamento objetivo; es obvio, nada hay en las características del objeto natural que nos lleve necesariamente a llamarle "árbol" ("tree"), y no existe ninguna semejanza entre el objeto y la palabra. La existencia y el uso de las palabras (incluidas aquellas que se utilizan para juzgar a los objetos) dependen entonces de una convención culturalmente definida (p. 94).

En consecuencia, es inútil buscar una ética objetivamente fundamentada. Por objetividad nos referimos a las características materiales de los objetos y a lo que Wittgenstein (2009) llama "los hechos del mundo". Al decir que la ética no posee un fundamento objetivo, aseveramos que los juicios éticos, morales o estéticos, no pueden ser inferidos de modo directo de los 
hechos del mundo. Sostendremos que para entender dichos juicios, tenemos que entrar en el mundo de lo cultural y, por lo tanto, del lenguaje y sus signos arbitrarios. Cabe aclarar que negar la objetividad de los juicios éticos no es negar la existencia de los objetos o la materialidad del mundo, sino manifestar que no basta con conocerlos a la perfección para construir un sistema ético.

Por otra parte, veremos que no se trata de una investigación sobre el bien, como lo planteó el primer Wittgenstein, sino sobre los juicios de valor que se expresan de distintas maneras, con variadas palabras y en diferentes circunstancias. Es así como, en una segunda parte del presente artículo, llegaremos a analizar las formas a través de las cuales los sujetos expresan sus valoraciones en situaciones concretas de interacción social.

\section{La ética para el "primer Wittgenstein"}

Con el término "ética", Wittgenstein no alude al conjunto de normas que rigen la convivencia social, sino a concepciones sobre el bien último y el sentido de la vida (Zavadivker, 2010), las cuales no son expresables con el lenguaje. En el parágrafo 6.421 del Tractatus (Wittgenstein, 2009), el filósofo lo afirma: "Está claro que la ética no resulta expresable. La ética es trascendental. (Ética y estética son una y la misma cosa.)". Esta declaración parte de una definición (particular) del lenguaje como representación del mundo. Como Zavadivker (2010, p. 3) lo dice, en el Tractatus se plantea un "isomorfismo entre lenguaje y mundo", ya que "una proposición puede trazar una figura de un hecho en virtud de compartir una forma común" (p. 3), y a cada palabra (genuina) le debe corresponder un objeto.

En el Tractatus (§ 6.124), por ejemplo, se dice que "las proposiciones lógicas describen el armazón del mundo o, más bien, lo representan". El lenguaje con verdadero sentido $-\mathrm{o}$ verdaderamente significativo- es el que habla del mundo y sus hechos. Esta definición (y delimitación) del lenguaje ha sido criticada, ya que lo reduce a una de sus posibles dimensiones, haciendo que el único lenguaje legítimo o dotado de verdadero significado, sea "aquel que funciona como un conjunto de proposiciones de carácter representativo" (Nieto, 2013; cursivas del autor).

Por otro lado, Wittgenstein (2009) sostiene también que "[el] sentido del mundo tiene que residir fuera de él. En el mundo todo es como es y todo sucede como sucede; en él no hay valor alguno" (§ 6.41). Así pues, si en el mundo las cosas son como son y no tienen valor en sí mismas, y el lenguaje significativo solo puede hablar de los hechos del mundo y nada más, se sigue lógicamente que el lenguaje no puede expresar las cuestiones de la ética.

Wittgenstein no ignora que en el lenguaje natural las personas hablan de la ética, pero sus proposiciones no tienen verdadero significado, ya que carecen de referentes entre los hechos del mundo. Por otra parte, las personas generalmente están hablando de juicios de valor relativo. Así, en la "Conferencia sobre Ética" (Wittgenstein, 1965), su autor indica que el lenguaje puede expresar juicios como "este es el camino correcto", pero ello solo tiene validez si se aclara de antemano el criterio o estándar para considerarlo "correcto". Por ejemplo, es necesario señalar de antemano que se utilizará la palabra "correcto" para designar el camino que conduce con mayor rapidez de A hacia B. Acá se trata de un juicio de valor relativo, donde el lenguaje se emplea para describir los hechos (los lugares, las condiciones de los caminos, etc.) y la adecuada relación entre fines $\mathrm{y}$ medios.

Pero para el primer Wittgenstein, esto no sería relevante en un estudio sobre las cuestiones éticas, esto es, sobre los valores absolutos. Sería absurdo pretender que exista un camino correcto en un sentido absoluto, un camino que deba parecer correcto para todo el mundo, con 
independencia de los criterios y de los lugares a donde se quiere llegar. La ética remite justamente al bien absoluto, "pero tal absoluto no encuentra ningún acomodo en las redes del lenguaje" (Nieto, 2013, p. 55).

Aunque no se puede hablar de ética, Wittgenstein (1965) tampoco niega que ese sentimiento de un bien absoluto exista; de hecho, añade el autor, ese sentimiento merece todo su respeto. Ese bien absoluto es algo trascendente, es decir, que trasciende los hechos del mundo; es "metafísico" por cuanto va más allá del mundo físico. De cierta manera, lo anterior se relaciona también con lo místico: "lo inexpresable, ciertamente, existe. Se muestra, es lo místico" (Wittgenstein, 2009, § 6.522).

Sin embargo, cuando alguien pretende decir algo de carácter metafísico, Wittgenstein querría "demostrarle que no ha dado significado a ciertos signos en sus proposiciones" ( $§ 6.53)$, puesto que su palabras no apuntan a los objetos o hechos del mundo. Lo místico no puede decirse con el lenguaje, "pues nada representa" (Nieto, 2013). La noción de bien absoluto o de lo absolutamente valioso existe porque los seres humanos la experimentan, la viven. Pero si no surge del mundo mismo y sus hechos, debe surgir fuera de él: "la ética, de ser algo, es sobrenatural" (Wittgenstein, 1965, p. 5).

Para el primer Wittgenstein, hablar de ética es arremeter contra los límites del lenguaje, lo cual puede entenderse como un señalamiento de las limitaciones que tiene un lenguaje que solo sirve para describir los hechos del mundo, y no puede entonces referirse a lo trascendental o a lo místico. Arremeter contra estos límites puede ser "un intento de extender y visibilizar un discurso perseguido y reprimido" (Soto, 2010 , p. 110) en un contexto sociocultural e histórico en el que el discurso del positivismo científico dominaba.

Por otra parte, al conectar la ética con lo metafísico o lo sobrenatural, "no se trataba de buscar divinidades u otras entidades supramundanas, sino más bien de superar posiciones 'naturalistas"' (Nieto, 2013, p. 49; cursivas del autor) en las discusiones sobre ética. Como veremos más adelante, ese naturalismo es precisamente lo que permite -aún hoy en día- hablar de "derecho natural" o legitimar juicios éticos a través de estudios científicos sobre la biología humana o la ecología.

\section{La diferencia entre razones y causas}

En el lenguaje común, y de hecho muchas veces en el lenguaje académico, se emplean las palabras "razones" y "causas" indistintamente. Sin embargo, para el segundo Wittgenstein, ese uso indiscriminado trae graves confusiones. Añadiremos luego que la diferenciación analítica entre los dos conceptos también arroja luces a propósito de las posibles explicaciones de los juicios éticos.

En Los cuadernos azul y marrón (Wittgenstein, 1968) se explica que tanto las razones como las causas responden a la pregunta "por qué", pero en sentidos muy diferentes. Las causas de un fenómeno se plantean como una hipótesis cuya validez se puede demostrar o invalidar a través de un cierto número de experimentos que, "hablando toscamente, concuerden en mostrar que la acción es la secuela regular de ciertas condiciones, que entonces llamamos causas de la acción" (p. 42). En cambio, si queremos "conocer la razón que se tuvo para hacer un cierto enunciado, para actuar de un modo determinado, etc. no se necesita ningún número de experiencias [o experimentos]" (p. 43) como los mencionados a propósito de las causas.

Las razones, al contrario de las leyes naturales (que encuentran la biología o la física), pueden variar según las convenciones culturales y los lenguajes, de modo que no podremos hallarlas a partir de experimentos. En cambio, "dar una razón es como dar una operación de cálculo mediante la cual se ha llegado a un cierto resultado" (p. 43). Así por ejemplo, las causas 
que se buscan en cierto tipo de experimentos psicológicos no permiten entender realmente las valoraciones estéticas; las causas, en ese tipo de experimentos, se refieren a los hechos fisiológicos que ocurren dentro de una persona cuando esta emite un juicio.

Las razones se construyen a través del lenguaje y buscan explicar, por ejemplo, la inclinación de un sujeto a creer que una combinación de colores o una serie de comportamientos son "correctas". Este tipo de explicación evoca las reglas de un lenguaje que permitieron manipular símbolos de una cierta manera, como en el caso de la operación de cálculo referida antes.

La idea de que juicios éticos puedan basarse en las propiedades naturales del mundo (causas) y no en los designios cambiantes de las sociedades (razones), lleva a creer erradamente que explicar por qué un comportamiento es "correcto" dependería de la "naturaleza humana" o sería algo similar a esclarecer por qué una sustancia reacciona de cierto modo con otra. Rechazando este tipo de analogías, Wittgenstein (1992, p. 97) demuestra que utilizamos de forma equívoca el lenguaje de la causalidad del mundo físico para hablar del mundo de la cultura. Por ejemplo, decimos que lógicamente "una doble negación produce una afirmación"”, al igual que decimos que "la combinación de dos sustancias produce ácido carbónico".

Pero la doble negación, insiste elfilósofo, no produce nada, no hay nada natural ${ }^{3}$ en el resultado de esa lógica, no hay nada en la naturaleza de la negación que conduzca inevitablemente a una afirmación cuando la primera se duplica. La doble negación simplemente se interpreta de cierta manera según la convención de una lógica particular usada en nuestra sociedad occidental.

Así mismo, no hay nada en la naturaleza de los objetos o los comportamientos que los haga buenos o inaceptables per se. ¿Qué hace que una combinación de colores sea sinónimo de mal gusto, o que un comportamiento sea in- aceptable? La cuestión remite a largos debates sobre la verdadera esencia de las cosas y el verdadero sentido de las palabras como "belleza" o "bondad", debates que aspiraban a definir, de una vez por todas, un fundamento sólido y definitivo para nuestros juicios: si antes era Dios la fuente de esas verdades éticas, ahora muchos piensan que debería ser la objetividad de la ciencia. Pero no es así.

Cabe señalar que la arbitrariedad de los sistemas éticos o estéticos no es sinónimo de caos o azar. Existen criterios ${ }^{4}$ sobre los cuales se define lo que es "correcto" o "apropiado", al igual que existen códigos culturales que nos indican cuándo aceptar o rechazar una cierta configuración de colores o un cierto comportamiento. Aunque no sean universales ni objetivos, dichos criterios y códigos seguramente han sido construidos luego de cientos de años de interacción humana, están apoyados por sólidas instituciones y complejos sistemas de valores, y son integrados a lenguajes y a prácticas económicas.

En el momento histórico en que Wittgenstein argumentara sobre la diferencia entre causas y razones, abogando por la importancia de las segundas, la comunidad científica veía con recelo todo lo que no pudiera ser explicado de la misma forma en que se explican los hechos físicos o químicos. Al respecto, Ayer (1976) se pregunta: "What is there about our belief in the physical world that makes it sacrosanct?". ¿Por qué las causas tienen una jerarquía superior a las razones a la hora de explicar un fenómeno humano? (p. 233).

\section{EI naturalismo de las causas y su posición dominante}

Si el lenguaje no es necesariamente representación de los objetos del mundo, tampoco lo es la expresión de "contenidos mentales". Wittgenstein también critica este concepto, considerándolo poco claro y "nebuloso" (Holborow, 1976). Nos dice, en cambio, que el significado está en el lenguaje mismo: "pregun- 
témonos qué es lo que explica la explicación de un significado, pues lo que esto explique será el significado" (Wittgenstein, 1968, p. 27). El filósofo señala además los muy diversos usos de la palabra "bello" (Wittgenstein, 1989), los cuales no necesariamente tienen un significado común o "de fondo" (no hay nada en común entre un bello día y un bello vestido, o entre un comportamiento correcto y un traje correcto).

Si preguntamos a alguien cuál fue el pensamiento que tuvo al pronunciar la frase (de carácter ético) "el voto debe ser un derecho universal", nos responderá que pensó justamente en esa frase, compuesta por esas palabras, sin podernos señalar un contenido mental al cual se refiere. ¿Cómo explicar el significado de esa frase si no es precisamente con una explicación de su significado? Y de nada sirve, añade Wittgenstein, recurrir al concepto de "imágenes mentales", porque estas tampoco tienen sentido en sí mismas.

La verdad es que una imagen, sea mental o no, no tiene ningún sentido o significado de manera universal o "natural": ella sería también un objeto de múltiples interpretaciones posibles. Tampoco soluciona las cosas decir que los contenidos mentales son como "sombras" de los objetos reales, ya que nos enfrentaríamos a otras dificultades, como por ejemplo la pregunta: "¿cómo puede algo ser la sombra de un hecho que no existe?" (Wittgenstein, 1968, p. 61).

Ahora bien, no es procedente hablar de nociones o ideas del bien o del mal que estarían en nosotros como contenidos mentales. ¿Cuál podría ser la "imagen" o el "contenido mental" que tenemos en la cabeza cuando pensamos en "lo correcto", sabiendo que un comportamiento correcto y un traje correcto no tienen nada en común? Y puede que, dentro de su pensamiento abstracto, un individuo tenga una imagen que se relaciona con las palabras "lo correcto" (digamos, una imagen de Jesús impartiendo luz con su mano), pero ciertamente no es gracias a ella que podemos decidir qué es correcto en las innumerables y muy distintas situaciones de la vida cotidiana.
En Los cuadernos azul y marrón (1968), el filósofo plantea que hablar del pensamiento como una actividad mental produce confusiones. En consecuencia, no debemos asumir como un hecho que el pensamiento -en tanto que manipulación de símbolos- tiene lugar en la cabeza o en el cerebro. "Si seguimos hablando sobre el lugar donde se realiza el pensamiento, tenemos derecho a decir que este lugar es el papel sobre el que escribimos [cuando pensamos escribiendo] o la boca que habla [cuando pensamos hablando]" (p. 33). Ubicar al lugar del pensamiento en nuestra cabeza es una expresión particular que debe ser entendida dentro de un lenguaje, y no como una descripción de los hechos por fuera de él.

A pesar de los señalamientos hechos por Wittgenstein hace varias décadas, se cree todavía que la ciencia ultramoderna y altamente tecnificada podría descubrir el origen fisiológico de los supuestos "contenidos mentales", para así explicarnos por qué pensamos lo que pensamos y por qué valoramos como valoramos.

Lo que se propone en la actualidad como "las verdaderas causas" del pensamiento tiende a remitir a reacciones físico-químicas que se llevan a cabo en el cerebro, todo lo cual se puede medir e incluso ver en pantallas de alta resolución. Así, se suele creer que el cerebro es el lugar donde se encuentran finalmente las verdaderas explicaciones sobre el comportamiento del ser humano (Vidal, 2009). Esta visión "neurocentrista" del hombre (Echarte \& Gaitán, 2013) se difunde y legitima cada día con más fuerza, y ello en buena parte debido a informaciones simplistas publicadas en los medios masivos de comunicación, en particular la prensa (en sus diferentes soportes) y los documentales televisados (Racine, Waldman, Rosenberg, \& Illes, 2010).

Se pretende así que procesos como percibir, amar, desear, elegir, apreciar o asombrarse deben entenderse únicamente en términos de causas cerebrales, es decir, fisiológicas. Gracias a esto ahora podemos ver en una pantalla la imagen cerebral que nos mostraría "por qué" alguien 
emite un juicio. Comprender al humano -en este difundido ideario- se reduce a conocer las conexiones neuronales, lo que implica utilizar el prestigioso lenguaje de las ciencias naturales o "exactas", y así tener la apariencia de poseer explicaciones más sólidas y válidas.

Aunque no dudamos que haya una cadena causal de conexiones sinápticas simultáneamente a la emisión de un juicio, es un error darle preponderancia a esta explicación, considerándola como "la razón de fondo" o lo que en últimas causa nuestros juicios. La mayor importancia y validez que se le otorga a ese tipo de explicación viene de "an inherited world-picture and of its propositions as being part of a kind of mythology" (Wittgenstein citado por Ayer, 1976, p. 234). La visión neurocentrada del hombre se legitima entonces por toda una visión del mundo y una suerte de mitología moderna.

Para Wittgenstein parte del error en las investigaciones sobre el pensamiento consiste en "creer que pensamos con nuestras cabezas, dentro de nuestras cabezas" (citado por Holborow, 1976; traducción propia). Si no pensamos con nuestra cabeza, ni dentro de ella, es absurdo limitar la explicación de nuestros pensamientos y juicios éticos o estéticos a un órgano físico y delimitado como el cerebro. Llegar a considerar que un determinado comportamiento es "mezquino" o "noble" es producto de un proceso complejo donde las conexiones neuronales son condiciones necesarias mas no suficientes.

De manera similar, para entender por $q u e ́$ un jugador de ajedrez realiza unas movidas particulares, las causas las podemos encontrar en el mundo físico (sus neuronas, los músculos de su mano que mueven las fichas, etc.), pero las razones de dichas movidas las encontraremos en las reglas del juego de ajedrez y en la disposición de las fichas en el tablero, en un momento dado.

En la medida en que Wittgenstein nos invita a poner en duda que la cognición sea algo que ocurre únicamente en nuestras cabezas (Susswein \& Racine, 2009), también podemos dudar de que las apreciaciones éticas o estéticas provengan simplemente de una parte de nuestro cuerpo. Para responder a esa duda, tendremos que dar cuenta del contexto o situación de interacción social, de las reglas de uso de las palabras en un juego particular que se desarrolla en esa situación, e incluso preguntarnos por el origen histórico y el proceso social complejo que dio origen a la situación de interacción y a las reglas del juego, todo lo cual va mucho más allá del cerebro, y mucho más allá de un solo individuo.

\section{Los juicios de valor: de las esencias a las reglas de un juego}

Claramente, los juicios de valor no están en el mismo nivel epistemológico que las proposiciones asertóricas. Sobre los primeros, debemos entender el lenguaje del que forman parte, comprender su gramática. El segundo Wittgenstein nos invita a comenzar por el estudio de los juicios tal como se manifiestan en sociedad, lo que ocurre precisamente a través del lenguaje, mas no en un lenguaje ideal y restringido a su capacidad representativa, sino en el lenguaje como medio de comunicación en las relaciones sociales cotidianas, por lo que es necesario considerar las maneras en que los sujetos emplean las palabras, lo que hacen con ellas, la "forma de vida" en que se inscriben (Wittgenstein, 1999, pp. 12-15).

Para el segundo Wittgenstein, el lenguaje no es tan solo una forma de descripción del mundo, sino más bien una caja de herramientas que se usa para interactuar socialmente y para construir realidades; cada palabra es como una herramienta particular que aprendemos a utilizar en determinadas situaciones de la vida cotidiana, situaciones en donde se establecen ciertos "juegos de lenguaje" (pp. 10-17). El significado de una palabra no necesariamente se refiere a un objeto o hecho del mundo. Para entender el significado de una palabra, es clave discernir su uso en contextos particulares (p. 21).

Lo anterior podemos aplicarlo a la comprensión de los términos con los que expresa- 
mos juicios éticos o estéticos, recordando que "no nos concentraremos en las palabras 'bueno' o 'bello', que no son nada características, generalmente solo sujeto y predicado ('esto es bello')" (p. 65). En cambio, analizaremos el uso de palabras como "incoherente", "preciso", "apropiado", e incluso notaremos el empleo de algunos gestos de la cara, que pueden reemplazar ciertas palabras (p. 66). Podemos entonces observar una gran variedad de palabras, pero nos concentraremos en especial en "las ocasiones en las que se dicen" (p. 66), ocasiones en las cuales, como ya se mencionó, se llevan a cabo determinados juegos de lenguaje.

El utilizar la palabra "correcto" cuando se trata de un traje o de un comportamiento, depende de las reglas de uso de esa palabra (o de un gesto) en situaciones muy diferentes. Las palabras se emplean según las reglas de un juego de lenguaje, así como se usan las fichas en un juego de ajedrez. A propósito de esta comparación, el filósofo afirma: "La pregunta '¿Qué es realmente una palabra?' es análoga a ‘¿Qué es una pieza de ajedrez?"” (p. 43). Por ejemplo, un juego de lenguaje se desarrolla en la situación de elección de un traje, y otro juego de lenguaje se aplica al juzgar los comportamientos en una sala de cine. En cualquier caso, se trata de llevar a cabo evaluaciones (Wittgenstein, 1989, p. 70), en las que comparamos un estado de cosas particular con respecto a un criterio o un patrón (como es claro en el caso del traje que consideramos "correcto"). Frente a una catedral gótica, nos dice el filósofo, "el juego entero es diferente", y las palabras que usamos -aunque sean significantes iguales, como "bueno" o "correcto"- desempeñan un papel por completo distinto (p. 71).

Los planteamientos de Wittgenstein contradicen en varios aspectos a las teorías tradicionales sobre el lenguaje que se encuentran ya desde San Agustín en el siglo IV ${ }^{5}$. Las palabras de una lengua -se solía y se suele pensar- tienen un significado, el cual no es otra cosa que un objeto que representan. Dicho de otra forma, las palabras nombran objetos. Wittgenstein claramente critica esta noción del lenguaje relacionada con lo que Feyerabend llamó "realismo medieval" y un cierto "esencialismo" (Feyerabend, 1955, p. 449), y aclara que usar el lenguaje para nombrar objetos es solo uno de los muchos juegos de lenguaje que los humanos pueden inventar (Wittgenstein, 1999, p. 17). Desde esa tradición medieval se cree que unos lenguajes pueden ser más perfectos que otros, ya que denotarían de modo más preciso la verdadera esencia de los objetos.

Casi en dirección opuesta, hoy en día se ha confirmado (Boroditsky, 2011) que el lenguaje influencia nuestra cognición, nuestra percepción, nuestra visión del mundo (por ejemplo la manera en que concebimos el espacio y el tiempo), la manera como lo dividimos en categorías y objetos, la forma en que recordamos, las diferenciaciones que establecemos, las cualidades que les atribuimos, todo lo cual incide en nuestras preferencias y juicios.

La vida social está compuesta de múltiples y heterogéneos juegos de lenguaje a través de los cuales aprendemos a distinguir los objetos, a clasificarlos y a vincularlos a ciertas calidades y responder ante ellos de ciertas maneras, tanto somática, como cognitiva y emocionalmente. En otras palabras, es mediante los juegos, tal como Wittgenstein entiende el concepto, que aprendemos a emitir juicios de todo tipo, lo cual constituye, por supuesto, el sistema ético.

En este mismo sentido, François Lyotard, quien también maneja sistemáticamente el concepto de "juegos de lenguaje", considera que la política crítica es una reflexión en función de un juego de lenguaje especial llamado "ser justo" (Lyotard citado por Vega, 2010, p. 31). Así mismo, podemos decir que las valoraciones éticas constituyen una clase peculiar de juego cuyo fin es usar cierto tipo de calificativos frente a distintas situaciones de la vida, en especial cuando se trata de comportamientos humanos.

Los juicios de valor (de los cuales dependen las leyes del derecho o las decisiones polí- 
ticas) se articulan también con construcciones ontológicas, es decir, con definiciones de lo que es. Por ejemplo, si se afirma que "esto es un homicidio", esa definición surge de una cosmovisión, de unos criterios de clasificación de los seres vivos, de unas reglas de uso de las palabras. Las definiciones suelen implicar valoraciones. Se trata en buena parte de un conocimiento tácito que por lo general se estima como una simple cuestión de "sentido común", algo que se da por sentado. Todo ello surge al interior de un vasto sistema cultural en el que los elementos se precisan en relación con los demás y forman parte de un modo de vida, porque todo juego de lenguaje está entretejido con una serie de acciones o prácticas (Wittgenstein, 1999, p. 10).

Las reglas de juegos son -evidentementeproducto de la voluntad humana y, por lo tanto, podrían ser otras. Sin embargo, esto no implica que se trate de banalidades efímeras. De hecho, la introducción de cambios en los sistemas culturales nunca ha sido un proceso simple y rápido. Incluso en el caso de un juego como el ajedrez, un cambio en sus reglas implicaría vencer no pocas dificultades: decidir, por ejemplo, que la ficha del alfil es indestructible, sería una modificación incompatible con otras reglas del juego y con el fin mismo de este (que es vencer a un contrincante eliminando todas sus fichas).

Nuestro reformador del juego tendría que argumentar sus razones con la comunidad de jugadores de ajedrez, y muchos dirían que no tendría sentido hacerle semejante cambio al juego, o dirían simplemente que ese sería otro juego al cual seguramente no querrían jugar. Cabe recordar acá que en función de los múltiples juegos de lenguaje que aprendemos en sociedad, se define el carácter deseable, noble, correcto, necesario, u otro, de los hechos del mundo, razón por la que las personas reaccionan y se movilizan, se alegran o se hacen la guerra. Los juegos implican prácticas, por lo que introducir modificaciones en ellos conllevaría lograr también la aceptación y adaptación por parte de la comunidad y sus instituciones.

\section{Conclusiones}

Hemos visto que las reflexiones de Ludwig Wittgenstein permiten entender que no es pertinente buscar las causas de la belleza o la bondad. En cambio, nuestros objetos de análisis deben ser, por un lado, las expresiones reales de las personas en sus juicios particulares $y$, por otro, las razones y el sistema cultural que validan dichas expresiones. Al evacuar una discusión sobre las causas objetivas de una ética (causas que estarían en el mundo de los objetos o en la naturaleza del cerebro), tenemos que cuestionar el naturalismo que ello implica $\mathrm{y}$, por ende, cuestionar también importantes nociones como la del "derecho natural" (de la cual surge el concepto de "derechos humanos").

Si bien en un proceso judicial puede ser necesario establecer la causalidad de ciertos hechos, no podemos seguir asumiendo que estos hablan por sí solos o tienen valor en sí mismos, ya que tanto su definición y clasificación, como las razones sobre las que reposa finalmente un veredicto, son producto de la voluntad humana, es decir, son arbitrarias (en el sentido que hemos utilizado aquí). Ningún juicio es entonces universalmente válido con el argumento de que obedece tan solo a la naturaleza de los hechos del mundo.

Esta conclusión puede incomodar, ya que con frecuencia se piensa que sin un fundamento objetivo o natural, quedaremos perdidos en un universo de anomia y caos social. Semejante temor quizá se agudiza en una modernidad tardía donde la desinstitucionalización y la desorientación del individuo se profundizan, lo cual genera búsquedas compulsivas de certezas y principios estables (Bauman, 2002, p. 26). Un supuesto fundamento objetivo podría aportar tal certeza y estabilidad ${ }^{6}$. No obstante, se trata de una falacia peligrosa, por cuanto sirve también para que algunos grupos sociales crean que poseen la clave para determinar de manera incuestionable lo que es bueno y lo que es malo, y de ahí, pretendan definir cómo se debe comportar el resto del mundo, sometiéndolo. 
Si entre nosotros ciertas razones se consideran como más válidas que otras (el ejercicio del derecho se basa en esa suposición), es importante reconocer que ello es parte de un juego particular de la cultura occidental, que se corresponde con una forma de vida y con una visión del mundo específicas.

Posiblemente cueste trabajo creer que los juicios y principios que nos parecen "naturales" y se articulan con hondos sentimientos de ira, amor, lealtad, etc., se cimienten en juegos aprendidos en sociedad, cuyas reglas son arbitrarias. Pero hay que recordar que los juegos se instituyen y adquieren legitimidad social. Es un error confundir la sólida fundamentación de las razones con la objetividad de las causas, o pretender que sin un soporte objetivo, los principios éticos o morales carecen de legitimidad o realidad para los individuos.

Las reglas de los juegos y las razones que sustentan los juicios éticos forman parte de los sistemas culturales humanos. Si podemos afirmar que la vida social es un conjunto de juegos, debemos recordar que estos implican prácticas y tienen efectos muy reales y concretos en el mundo material. Así mismo, las reglas de estos juegos definen la comunicación y la organización social, política y económica de las sociedades, que han sido claves para nuestra supervivencia misma como especie.

Infortunadamente, decir que los principios éticos están entera y directamente apoyados en las características de los hechos del mundo, se ha convertido en una manera de justificar la descalificación de otros sistemas éticos, lo que también ha dado pie, en muchos casos, al colonialismo y a la exterminación de la diferencia cultural. Así, la reflexión a la que nos conduce Wittgenstein permite también reconocer la pluralidad de los juicios de valor y aceptar que el Otro tiene razones tan válidas como las propias para juzgar y actuar.

Recordar el fundamento arbitrario (en el sentido en que se explicó la palabra) de los juicios éticos no es sinónimo de defender el "todo-vale posmoderno" del que habla Lyotard (1979). Es claro que una sociedad necesita unas reglas y una cierta forma de orden social. Sin embargo, una cosa es buscar un orden sólidamente justificado (con razones válidas dentro de un sistema cultural), y otra cosa muy distinta es pretender naturalizarlo y, de ahí, imponerlo universalmente.

\section{Notas}

${ }^{1}$ Con esta expresión se designa el trabajo del filósofo en una primera etapa de su vida, trabajo que contrasta con el de la segunda etapa, o el "segundo Wittgenstein".

${ }^{2}$ Un ejemplo de una doble negación es "no creo que sea imposible", la cual se lee como la afirmación "creo que es posible".

${ }^{3}$ El concepto de lo "natural" se entiende aquí por contraste con las construcciones histórico-culturales de la humanidad, como es el caso de los lenguajes con sus reglas y signos arbitrarios.

${ }^{4}$ Sobre la importancia de la noción de “criterio" en Wittgenstein, véase Albritton (1968).

${ }^{5}$ San Agustín se refirió al lenguaje en casi todas sus obras (véase la compilación: Agustín, 1963).

${ }^{6}$ Un imaginario fundamento divino también lograría tales efectos $\mathrm{y}$, sin embargo, permitir a los fundamentalistas religiosos definir la política y las leyes es impensable en nuestra modernidad.

\section{Referencias}

Agustín, Santo, Obispo de Hipona. (1963). Obras de San Agustín. Madrid: Editorial Católica.

Albritton, R. (1968). On Wittgenstein's Use of the Term 'Criterion'. En G. Pitcher (Ed.), 
Wittgenstein. The Philosophical Investigations (pp. 34-59). Indiana: University of Notre Dame.

Ayer, A. J. (1976). Wittgenstein on Certainty. En G. Vesey (Ed.), Understanding Wittgenstein (pp. 226-245). Ithaca: Cornell University Press.

Bauman, Z. (2002). Modernidad Líquida. Buenos Aires: Fondo de Cultura Económica.

Boroditsky, L. (2011). How Language Shapes Thought. Scientific American, 304, 62-65.

Echarte, L., \& Gaitán, L. (2013). Causas emocionales y dinámicas de las actuales expectativas en Neurociencia. Pensamiento y Cultura, 16(2), 8-32.

Feyerabend, P. (1955). Wittgenstein's Philosophical Investigations. The Philosophical Review, 64(3), 449-483.

Holborow, L. (1976). Prejudice in Favour of Psychophysical Parallelism. En G. Vesey (Ed.), Understanding Wittgenstein (pp. 103-207). Ithaca: Cornell University Press.

Lyotard, J. F. (1979). La Condition Postmoderne. Rapport sur le savoir. Paris: Minuit.

Moore, G. E. (1983). Defensa del Sentido Común y Otros Ensayos. Barcelona: Ediciones Orbis.

Nieto, C. (2013). El arte de la vida. Wittgenstein en la encrucijada entre ética y estética. Valenciana, 11, 33-68.

Racine, E., Waldman, S., Rosenberg, J., \& Illes, J. (2010). Contemporary neuroscience in the media. Social Science \& Medicine, 71, 725-733.

Saussure, F. (1945). Curso de Lingüística General. Buenos Aires: Losada.
Soto, L. G. (2010). Sobre Wittgenstein y la Ética. Ágora, 29(1), 103-111.

Susswein, N., Racine, T. (2009). Wittgenstein and not-just-in-the-head cognition. New Ideas in Psychology, 27(2), 184-196.

Vega, A. (2010). Perspectivas de la estética y la política en J.F. Lyotard. Revista de Estudios Sociales, 35, 26-40

Vidal, F. (2009). Brainhood, anthropological figure of modernity. History of the Human Sciences, 22(1), 5-36.

Wittgenstein, L. (1965). Conferencia sobre Ética. The Philosophical Review, 74(1), 3-12. Recuperado de http://www.ddooss. org/articulos/textos/Wittgenstein_etica. pdf

Wittgenstein, L. (1968) Los Cuadernos Azul y Marrón. Madrid: Tecnos.

Wittgenstein, L. (1989). Lecciones y Conversaciones sobre Estética, Psicología y Creencia Religiosa. Barcelona: Paidós.

Wittgenstein, L. (1992). Gramática Filosófica. México D.F.: Universidad Nacional Autónoma de México.

Wittgenstein, L. (1999). Investigaciones Filosóficas. Barcelona: Altaya.

Wittgenstein, L. (2009). Tractatus LogicoPhilosophicus. Madrid: Alianza Editorial.

Zavadivker, N. (2010). Wittgenstein: ética y lenguaje. Espéculo. Revista de Estudios Literarios, 46. Recuperado de http:// www.ucm.es/info/especulo/numero46/ witetilen.html 
